\title{
Approaching Unit Visibility for Control of a Superconducting Qubit with Dispersive Readout
}

\author{
A. Wallraff, D. I. Schuster, A. Blais, L. Frunzio, J. Majer, S. M. Girvin, and R. J. Schoelkopf \\ Departments of Applied Physics and Physics, Yale University, New Haven, CT 06520
}

(Dated: October 25, 2018)

\begin{abstract}
In a Rabi oscillation experiment with a superconducting qubit we show that a visibility in the qubit excited state population of more than $90 \%$ can be attained. We perform a dispersive measurement of the qubit state by coupling the qubit non-resonantly to a transmission line resonator and probing the resonator transmission spectrum. The measurement process is well characterized and quantitatively understood. The qubit coherence time is determined to be larger than $500 \mathrm{~ns}$ in a measurement of Ramsey fringes.
\end{abstract}

One of the most promising solid-state architectures for the realization of a quantum information processor [1] is based on superconducting electrical circuits [2]. A variety of such circuits acting as qubits [1], the basic carriers of quantum information in a quantum computer, have been created and their coherent control has been demonstrated [3, 4, 5, 6, 7, 8]. Recent experiments have realized controlled coupling between different qubits [9, 10, 11, 12, 13] and also first two-qubit quantum logic gates [14].

An outstanding question for superconducting qubits, and in fact for all solid-state implementations of quantum information processors, is whether the qubits are sufficiently well-isolated to allow long coherence times and high-fidelity preparation and control of their quantum states. This question is complicated by the inevitable imperfections in the measurement. A canonical example is a Rabi oscillation experiment, where the experimenter records the oscillations of a meter's response as a function of pulse length to infer the qubit's excited state population immediately after the pulse. The measurement contrast (e.g. the amplitude of the meter's measured swing relative to its maximum value) is reduced in general by both errors in the qubit preparation and readout, and sets only a lower limit on the visibility of oscillations in the qubit population. Most experiments with superconducting qubits to date have reported only the measurement contrast, typically in the range of $10-50 \%$ [3, 4, [5, 6, 7, 8, 14].

A full understanding of the measurement process is required to extract the qubit population from the meter's output. The qubit control and read-out is then characterized by the visibility, defined as the maximum qubit population difference observed in a Rabi oscillation or Ramsey fringe experiment. Moreover, it is essential to demonstrate that a qubit can be controlled without inducing undesired leakage to other qubit states or entanglement with the environment. We note that experiments have suggested that there can be substantial reduction of the visibility due to entanglement with spurious environmental fluctuators [15].

In this letter, we report results on time-domain con- trol of the quantum state of a superconducting qubit, where the qubit state is measured using a dispersive microwave measurement in a circuit quantum electrodynamics (QED) architecture [16]. This novel technique has shown good agreement with predictions in steady-state experiments 17]. Here, we observe the measurement response, both during and after qubit state manipulation, which is in quantitative agreement with the theoretical model of the system, allowing us to separate the contributions of the qubit and the readout to the observed contrast. The observed contrast of $85 \%$ and a visibility of greater than $95 \%$ for Rabi oscillations demonstrates that high accuracy control can be achieved in superconducting qubits.

In our circuit QED architecture [16], a Cooper pair box [18], acting as a two level system with ground $|\downarrow\rangle$ and excited states $|\uparrow\rangle$ and level separation $E_{a}=\hbar \omega_{a}=$ $\sqrt{E_{\mathrm{el}}^{2}+E_{\mathrm{J}}^{2}}$ is coupled capacitively to a single mode of the electromagnetic field of a transmission line resonator with resonance frequency $\omega_{\mathrm{r}}$, see Fig. 19. As demonstrated for this system, the electrostatic energy $E_{\mathrm{el}}$ and the Josephson energy $E_{\mathrm{J}}$ of the split Cooper pair box can be controlled in situ by a gate voltage $V_{g}$ and magnetic flux $\Phi$ [17, 19], see Fig. [1. In the resonant $\left(\omega_{a}=\omega_{r}\right)$ strong coupling regime a single excitation is exchanged coherently between the Cooper pair box and the resonator at a rate $g / \pi$, also called the vacuum Rabi frequency [19]. In the non-resonant regime $\left(|\Delta|=\left|\omega_{a}-\omega_{r}\right|>g\right)$ the capacitive interaction gives rise to a dispersive shift $\left(g^{2} / \Delta\right) \sigma_{z}$ in the resonance frequency of the cavity which depends on the qubit state $\sigma_{z}$, the coupling $g$ and the detuning $\Delta[16,17]$. We have suggested that this shift in resonance frequency can be used to perform a quantum non-demolition (QND) measurement of the qubit state 16]. With this technique we have recently measured the ground state response and the excitation spectrum of a Cooper pair box [17, 19].

In the experiments presented here, we coherently control the quantum state of a Cooper pair box by applying to the qubit microwave pulses of frequency $\omega_{s}$, which are resonant with the qubit transition frequency $\omega_{\mathrm{a}} / 2 \pi \approx 4.3 \mathrm{GHz}$, through the input port $C_{\text {in }}$ of the res- 
onator, see Fig. 1h. The microwaves drive Rabi oscillations in the qubit at a frequency of $\nu_{\mathrm{Rabi}}=\sqrt{n_{\mathrm{s}}} g / \pi$, where $n_{\mathrm{s}}$ is the average number of drive photons within the resonator. Simultaneously, we perform a continuous dispersive measurement of the qubit state by determining both the phase and the amplitude of a coherent microwave beam of frequency $\omega_{\mathrm{RF}} / 2 \pi=\omega_{\mathrm{r}} / 2 \pi \approx 5.4 \mathrm{GHz}$ transmitted through the resonator [16, 19]. The phase shift $\phi=\tan ^{-1}\left(2 g^{2} / \kappa \Delta\right) \sigma_{z}$ is the response of our meter from which we determine the qubit population. For the measurement, we chose a resonator that has a quality factor of $Q \sim 0.7 \times 10^{4}$ corresponding to a photon decay rate of $\kappa / 2 \pi=0.73 \mathrm{MHz}$. The resonator is populated with $n \sim 1$ measurement photons on average, where $n$ is calibrated using the ac-Stark shift [17].

We initially determine the maximum swing of the meter in a calibration measurement by first maximizing the detuning $\Delta$ to minimize the interaction $\left(g^{2} / \Delta \rightarrow 0\right)$ which defines $\phi=0$. Then, we prepare the Cooper pair box in the ground state $|\downarrow\rangle$ and bias it at charge degeneracy where the detuning is adjusted to $\Delta / 2 \pi \approx-1.1 \mathrm{GHz}$, corresponding to a maximum in the Josephson coupling energy of $E_{\mathrm{J}} / h \approx 4.3 \mathrm{GHz}<\omega_{\mathrm{r}} / 2 \pi$ for this particular

(a)

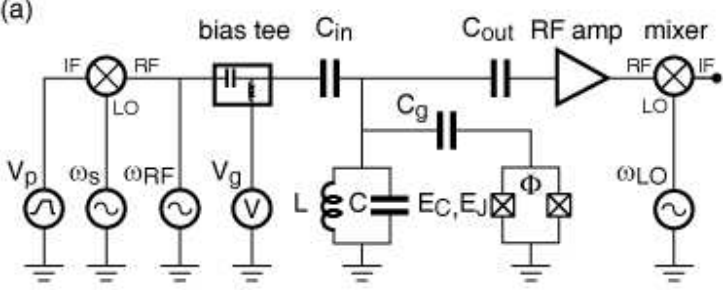

(b)

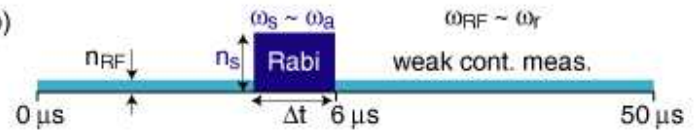

(c)

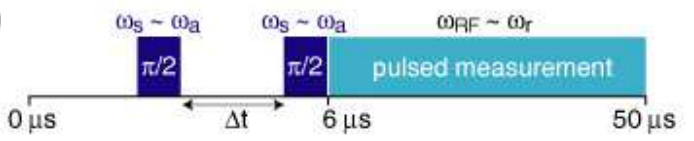

FIG. 1: (color online) (a) Simplified circuit diagram of measurement setup. A Cooper pair box with charging energy $E_{\mathrm{C}}$ and Josephson energy $E_{\mathrm{J}}$ is coupled through capacitor $C_{\mathrm{g}}$ to a transmission line resonator, modelled as parallel combination of an inductor $L$ and a capacitor $C$. Its state is determined in a phase sensitive heterodyne measurement of a microwave transmitted at frequency $\omega_{R F}$ through the circuit, amplified and mixed with a local oscillator at frequency $\omega_{\mathrm{LO}}$. The Cooper pair box level separation is controlled by the gate voltage $V_{g}$ and flux $\Phi$. Its state is coherently manipulated using microwaves at frequency $\omega_{\mathrm{s}}$ with pulse shapes determined by $V_{\mathrm{p}}$ [8]. (b) Measurement sequence for Rabi oscillations with Rabi pulse length $\Delta t$, pulse frequency $\omega_{\mathrm{s}}$ and amplitude $\propto \sqrt{n_{\mathrm{s}}}$ with continuous measurement at frequency $\omega_{\mathrm{RF}}$ and amplitude $\propto \sqrt{n_{\mathrm{RF}}}$. (c) Sequence for Ramsey fringe experiment with two $\pi / 2$-pulses at $\omega_{\mathrm{s}}$ separated by a delay $\Delta t$ and followed by a pulsed measurement. sample. In this case we measure a minimum meter response of $\phi_{|\downarrow\rangle}=-35.3 \mathrm{deg}$ corresponding to a coupling strength of $g / 2 \pi=17 \mathrm{MHz}$. Saturating the qubit transition by applying a long microwave pulse which incoherently mixes the ground and excited states such that the occupation probabilities are $P_{|\downarrow\rangle}=P_{|\uparrow\rangle}=1 / 2$, the measured phase shift is found to be $\phi=0$, as expected 17]. From these measurements, the predicted phase shift in the measurement beam induced by a fully polarized qubit $\left(P_{|\uparrow\rangle}=1\right)$ would be $\phi_{|\uparrow\rangle}=35.3 \mathrm{deg}$. Thus, the maximum swing of the meter is bounded by $\phi_{|\uparrow\rangle}-\phi_{|\downarrow\rangle}$.

In our continuous measurement of Rabi oscillations, the qubit is initially prepared in the ground state by relaxation, the thermal population of excited states being negligible. Then, a short microwave pulse of length $\Delta t$ prepares the qubit state while the measurement response $\phi$ is continuously monitored. This sequence is repeated every $50 \mu \mathrm{s}$, see Fig. 1 b, and the measurement response is digitally averaged $5 \times 10^{4}$ times. The signal to noise ratio (SNR) in the averaged value of $\phi$ in an integration time of $100 \mathrm{~ns}$ is approximately 25, see Fig. 2] corresponding to a SNR of 0.1 in a single shot. For the present experimental setup the single shot read-out fidelity for the qubit state integrated over the relaxation time $\left(T_{1} \sim 7 \mu \mathrm{s}\right)$ is

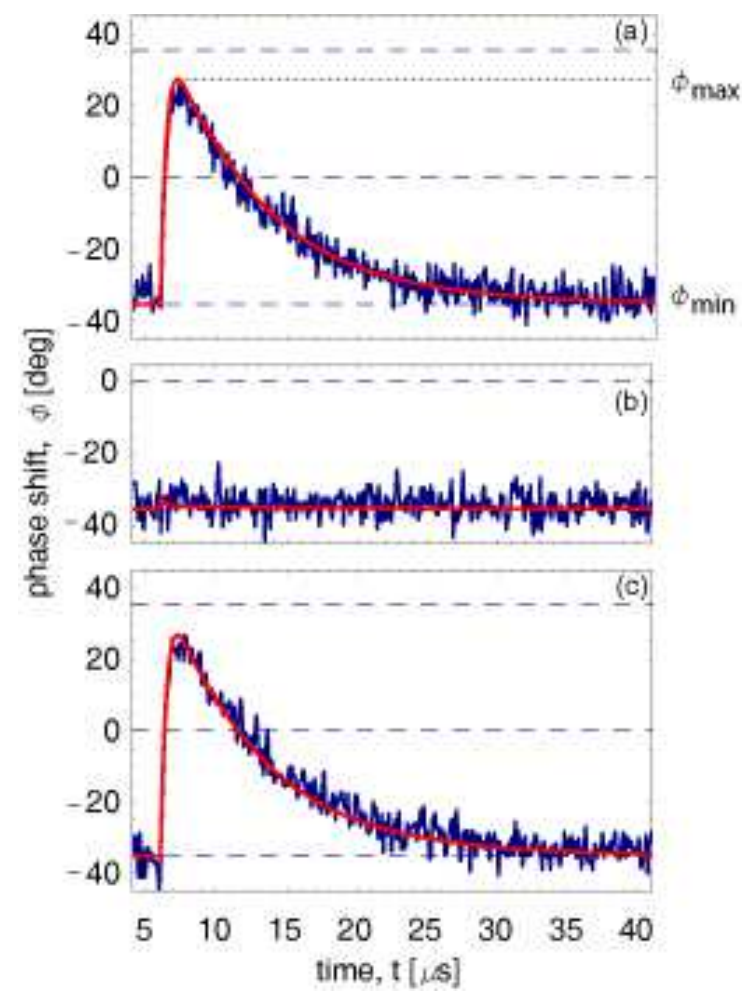

FIG. 2: (color online) Measurement response $\phi$ (blue lines) and theoretical prediction (red lines) vs. time. At $t=6 \mu \mathrm{s}$ (a) a $\pi$ pulse, (b) a $2 \pi$ pulse, and (c) a $3 \pi$ pulse is applied to the qubit. In each panel the dashed lines correspond to the expected measurement response in the ground state $\phi_{|\downarrow\rangle}$, in the saturated state $\phi=0$, and in the excited state $\phi_{|\uparrow\rangle}$. 
approximately $30 \%$ 20]. Both, a read-out amplifier with lower noise temperature or a larger signal power would potentially allow to realize high fidelity single shot measurements of the qubit state in this setup.

The time dependence of the averaged value of $\phi$ in response to a $\pi$ pulse of duration $\Delta t=1 / 2 \nu_{\text {Rabi }} \sim 16 \mathrm{~ns}$ applied to the qubit is shown in Fig. 22 . Before the start of the pulse the measured phase shift is observed to be $\phi_{|\downarrow\rangle} \approx-35.3 \mathrm{deg}$ corresponding to the qubit being in the ground state. Due to the state change of the qubit induced by the pulse, the resonator frequency is pulled by $2 g^{2} / \Delta$ and, thus, the measured phase shift is seen to rise exponentially towards $\phi_{|\uparrow\rangle}$ on the time scale of the intrinsic response time of the measurement $2 / \kappa \approx 400 \mathrm{~ns}$, i.e. twice the photon life time. At the end of the $\pi$ pulse, the qubit excited state decays exponentially on the time scale of its energy relaxation time $T_{1} \sim 7.3 \mu \mathrm{s}$, as extracted from the decay in the measured phase shift, see Fig. 21. As a result, the maximum measured response $\phi_{\max }$ does not reach the full value of $\phi_{|\uparrow\rangle}$. In general, the measurement contrast $C=\left(\phi_{\max }-\phi_{\min }\right) /\left(\phi_{|\uparrow\rangle}-\phi_{|\downarrow\rangle}\right)$ will be reduced in any qubit read-out for which the timescale of the qubit decay is not infinitely longer than the response time of the measurement. Additionally, in nonQND measurements the contrast is reduced even further due to mixing of the qubit states induced by the interaction with the measurement appartus. In our QND measurement presented here, the qubit decay time is about 15 times longer than the response time of the measurement, allowing us to reach a high maximum contrast of $C \sim 85 \%$ in the bare measurement response $\phi$.

In Figs. 20 and c, the measured response $\phi$ of the meter to a $2 \pi$ and a $3 \pi$ pulse acting on the qubit is shown. As expected, no phase shift is observable for the $2 \pi$ pulse since the response time of the resonator read-out is much slower than the duration $\Delta t=32 \mathrm{~ns}$ of the pulse. In agreement with the expectations for this QND scheme, the measurement does not excite the qubit out of its ground state, i.e. $\phi_{\min }=\phi_{\max }=\phi_{|\downarrow\rangle}$. The response to

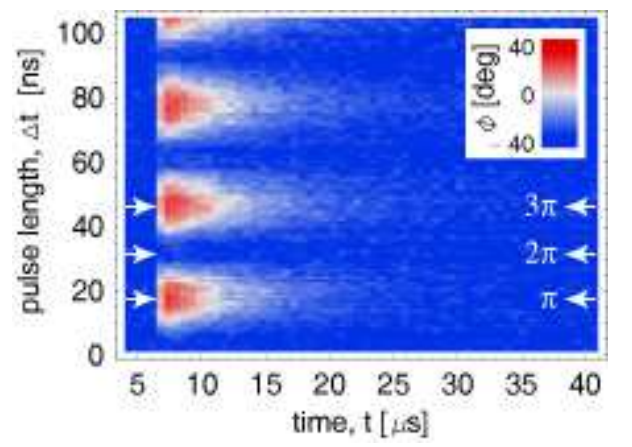

FIG. 3: (color online) Color density plot of phase shift $\phi$ (see inset for scale) versus measurement time $t$ and Rabi pulse length $\Delta t$. Data shown in Fig. 2 are slices through this data set at the indicated pulse lengths. the $3 \pi$ pulse is virtually indistinguishable from the one to the $\pi$ pulse, as expected for the long coherence and energy relaxation times of the qubit. In the $2 \mathrm{D}$ density plot Fig. 31 Rabi oscillations are clearly observed in the phase shift acquired versus measurement time $t$ and Rabi pulse length $\Delta t$.

The observed measurement response $\phi$ is in excellent agreement with the theoretical predictions, see red lines in Fig. 2] demonstrating a good understanding of the measurement process. The temporal response $\phi(t)=$ $\arg \{i\langle a(t)\rangle\}$ of the cavity field $a$ is calculated by deriving and solving Bloch-type equations of motion for the cavity and qubit operators [21] using the Jaynes-Cummings Hamiltonian in the dispersive regime [16, 17] as the starting point. A semi-classical factorization approximation is done to truncate the resulting infinite set of equations to a finite set (e.g. $\left\langle a^{\dagger} a \sigma_{z}\right\rangle \sim\left\langle a^{\dagger} a\right\rangle\left\langle\sigma_{z}\right\rangle$; all lower order products are kept). This amounts to neglecting higher order correlations between qubit and field which is a valid approximation in the present experiment. The calculations accurately model the exponential rise in the observed phase shift on the time scale of the resonator response time due to a state change of the qubit. They also accurately capture the reduced maximum response $\phi_{\max }$ due to the exponential decay of the qubit. Overall, excellent agreement in the temporal response of the measurement is found over the full range of relevant qubit and measurement time scales, see Fig. 22 All parameters used in the comparison of theory with experimental data have been extracted from independent measurements. The parameters remain fixed for all Rabi pulse length and thus leave no adjustables.

The visibility of the excited state population $P_{|\uparrow\rangle}$ in the Rabi oscillations is extracted from the time dependent measurement response $\phi$ for each Rabi pulse length $\Delta t$. We find $P_{|\uparrow\rangle}$ by calculating the normalized dot product (a)

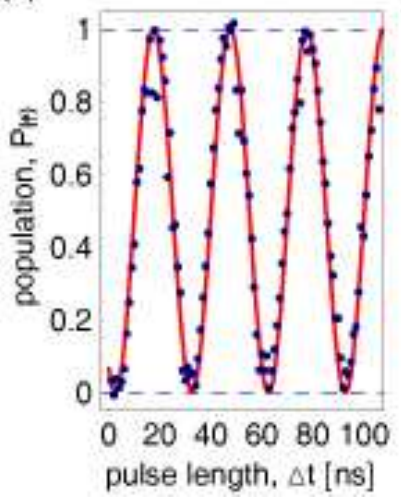

(b)

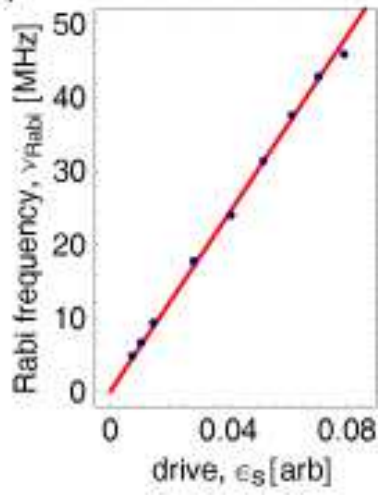

FIG. 4: (color online) (a) Rabi oscillations in the qubit population $P_{|\uparrow\rangle}$ vs. Rabi pulse length $\Delta t$ (blue dots) and fit with unit visibility (red line). (b) Measured Rabi frequency $\nu_{\text {Rabi }}$ vs. pulse amplitude $\epsilon_{\mathrm{S}}$ (blue dots) and linear fit. 
(a)

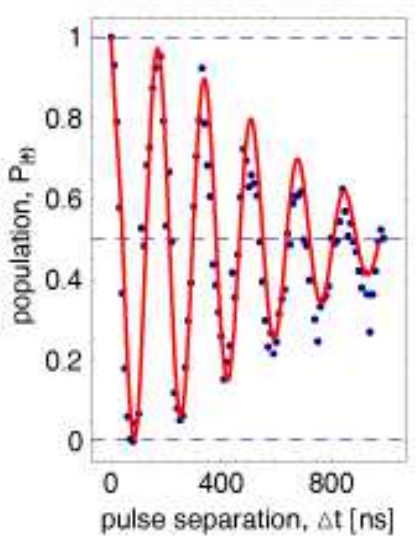

(b) detuning, $\triangle_{\mathrm{g}, \mathrm{s}}[\mathrm{MHz}]$



FIG. 5: (color online) (a) Measured Ramsey fringes (blue dots) observed in the qubit population $P_{|\uparrow\rangle}$ vs. pulse separation $\Delta t$ using the pulse sequence shown in Fig. 10 and fit of data to sinusoid with gaussian envelope (red line). (b) Measured dependence of Ramsey frequency $\nu_{\text {Ramsey }}$ on detuning $\Delta_{\mathrm{a}, \mathrm{s}}$ of drive frequency (blue dots) and linear fit (red line).

between the response $\phi$ and the theoretically predicted response taking into account the systematics of the measurement. This amounts to comparing the full area under a measured response curve, such as those shown in Fig. 2] to the theoretically predicted area. The averaged response of all measurements taken over a window in time extending from the start of the Rabi pulse out to several qubit decay times $T_{1}$ is used in our continuous dispersive measurement to extract $P_{|\uparrow\rangle}$. This maximizes the signal to noise ratio in the extracted Rabi oscillations.

The extracted qubit population $P_{|\uparrow\rangle}$ is plotted versus $\Delta t$ in Fig. 四. We observe a visibility of $95 \pm 6 \%$ in the Rabi oscillations with error margins determined from the residuals of the experimental $P_{|\uparrow\rangle}$ with respect to the predicted values. Thus, in a measurement of Rabi oscillations in a superconducting qubit, a visibility in the population of the qubit excited state that approaches unity is observed for the first time. Moreover, we note that the decay in the Rabi oscillation amplitude out to pulse lengths of $100 \mathrm{~ns}$ is very small and consistent with the long $T_{1}$ and $T_{2}$ times of this charge qubit, see Fig. 四 and Ramsey experiment discussed below. We have also verified the expected linear scaling of the Rabi oscillation frequency $\nu_{\text {Rabi }}$ with the pulse amplitude $\epsilon_{\mathrm{s}} \propto \sqrt{n_{\mathrm{s}}}$, see Fig. 4b.

We have determined the coherence time of the Cooper pair box from a Ramsey fringe experiment, see Fig. 11; when biased at the charge degeneracy point where the energy is first-order insensitive to charge noise [4]. To avoid dephasing induced by a weak continuous measurement beam 17] we switch on the measurement beam only after the end of the second $\pi / 2$ pulse. The resulting Ramsey fringes oscillating at the detuning frequency

$\Delta_{\mathrm{a}, \mathrm{s}}=\omega_{\mathrm{a}}-\omega_{\mathrm{s}} \sim 6 \mathrm{MHz}$ decay with a long coherence time of $T_{2} \sim 500 \mathrm{~ns}$, see Fig. 5 k. The corresponding qubit phase quality factor of $Q_{\varphi}=T_{2} \omega_{\mathrm{a}} / 2 \sim 6500$ is similar to the best values measured so far in qubit realizations biased at such an optimal point [4]. The Ramsey frequency is shown to depend linearly on the detuning $\Delta_{\mathrm{a}, \mathrm{s}}$, as expected, see Fig. 5b. We note that a measurement of the Ramsey frequency is an accurate time resolved method to determine the qubit transition frequency $\omega_{a}=\omega_{s}+2 \pi \nu_{\text {Ramsey }}$.

In conclusion, performing Rabi and Ramsey experiments we have observed high visibility in the oscillations of state population of a superconducting qubit. The temporal response and the back-action of the read-out are quantitatively understood and well characterized. Our charge qubit, which is embedded in a well controlled electromagnetic environment, has $T_{1}$ and $T_{2}$ times among the longest realized so far in superconducting systems. The simplicity and level of control possible in this circuit QED architecture makes it an attractive candidate for superconducting quantum computation.

We would like to thank Michel Devoret and Jay Gambetta for discussions. This work was supported in part by NSA and ARDA under ARO contract number DAAD1902-1-0045, and the NSF under grants ITR-0325580 and DMR-0342157, the David and Lucile Packard Foundation, the W. M. Keck Foundation, and the NSERC of Canada.

[1] M. A. Nielsen and I. L. Chuang, Quantum computation and quantum information (Cambridge University Press, 2000).

[2] M. H. Devoret, A. Wallraff, and J. M. Martinis, Superconducting qubits: A short review (2004), cond-mat/0411174 and references therein.

[3] Y. Nakamura, Y. A. Pashkin, and J. S. Tsai, Nature 398, 786 (1999).

[4] D. Vion et al., Science 296, 886 (2002).

[5] J. M. Martinis, S. Nam, J. Aumentado, and C. Urbina, Phys. Rev. Lett. 89, 117901 (2002).

[6] Y. Yu et al., Science 296, 889 (2002).

[7] I. Chiorescu, Y. Nakmura, C. J. P. M. Harmans, and J. E. Mooij, Science 299, 1869 (2003).

[8] E. Collin et al., Phys. Rev. Lett. 93, 157005 (2004).

[9] A. J. Berkley et al., Science 300, 1548 (2003).

[10] Y. A. Pashkin et al., Nature 421, 823 (2003).

[11] J. B. Majer et al., cond-mat/ 0308192 (2003), (submitted to Phys. Rev. Lett.).

[12] I. Chiorescu et al., Nature (London) 431, 159 (2004).

[13] R. McDermott et al., (2004), (submitted to Science).

[14] T. Yamamoto et al., Nature 425, 941 (2003).

[15] R. W. Simmonds et al., Phys. Rev. Lett. 93, 077003 (2004).

[16] A. Blais et al., Phys. Rev. A 69, 062320 (2004).

[17] D. I. Schuster et al., Phys. Rev. Lett. (2005), (in press, also: cond-mat/0408367).

[18] V. Bouchiat et al., Physica Scripta T76, 165 (1998).

[19] A. Wallraff et al., Nature (London) 431, 162 (2004).

[20] D. I. Schuster et al. (2005), unpublished. 
[21] A. Blais et al. (2005), unpublished. 